\title{
Polyphenol Extract of Moringa Oleifera Leaves Alleviates Colonic Inflammation in Dextran Sulfate Sodium-Treated Mice
}

\author{
Yunjuan Zhang, ${ }^{1}$ Lei Peng, ${ }^{2,3}$ Wenyun Li, ${ }^{1}$ Tianyi Dai, ${ }^{2,3}$ Long Nie, ${ }^{1}$ Jing Xie, ${ }^{2,3}$ Yu Ai, \\ Lingfei Li $\mathbb{D}^{1}{ }^{1}$ Yang Tian $\mathbb{D},{ }^{1,2,3}$ and Jun Sheng $\mathbb{D}^{4}$ \\ ${ }^{1}$ College of Food Science and Technology, Yunnan Agricultural University, Kunming 650201, China \\ ${ }^{2}$ Yunnan Provincial Key Laboratory of Biological Big Data, Yunnan Agricultural University, Kunming 650201, China \\ ${ }^{3}$ National R\&D Center for Moringa Processing Technology, Yunnan Agricultural University, Kunming 650201, China \\ ${ }^{4}$ Key Laboratory of Pu-er Tea Science, Ministry of Education, Yunnan Agricultural University, Kunming 650224, China \\ Correspondence should be addressed to Lingfei Li; lingfeili@163.com, Yang Tian; tianyang1208@163.com, \\ and Jun Sheng; shengjun_ynau@163.com
}

Received 30 July 2020; Revised 19 October 2020; Accepted 27 October 2020; Published 25 November 2020

Academic Editor: Armando Zarrelli

Copyright (C) 2020 Yunjuan Zhang et al. This is an open access article distributed under the Creative Commons Attribution License, which permits unrestricted use, distribution, and reproduction in any medium, provided the original work is properly cited.

\begin{abstract}
Moringa oleifera Lam. is an essential herb used for the treatment of inflammation, diabetes, high blood pressure, and other diseases. In this study, phenolic extracts of $M$. oleifera leaves were obtained and analyzed. The results showed that the main identifiable phenols were astragalin, chlorogenic acid, isoquercitrin, kaempferitrin, luteolin, quercetin, and rutin. The effects of M. oleifera polyphenol extract (MOPE) on experimental colitis induced by $3 \%$ dextran sulfate sodium (DSS) were investigated. The results showed that oral administration of MOPE significantly alleviated the symptoms of DSS-induced colitis. MOPE significantly reduced weight loss, the disease activity index, colon shortening, and mucosal damage. In addition, MOPE attenuated the infiltration of $\mathrm{CD}^{+}$T cells, $\mathrm{CD} 177^{+}$neutrophils, and F4/80 $0^{+}$macrophages and significantly inhibited the secretion of IL- 6 and TNF- $\alpha$. After the MOPE administration, the expression of proteins associated with the NF- $\kappa \mathrm{B}$ signaling pathway changed. Specifically, compared with that of the DSS group, the protein expression of NF- $\kappa \mathrm{B} p 65$ and $\mathrm{p}-\mathrm{I} \kappa \mathrm{B} \alpha$ was downregulated, and the expression of $\mathrm{I} \kappa \mathrm{B} \alpha$ was upregulated. This study revealed the anti-inflammatory effects and mechanisms of MOPE in the colon, indicating its potential use in preventing inflammation-driven diseases.
\end{abstract}

\section{Introduction}

Moringa oleifera Lam. has been historically used as nutritious food and traditional medicine in China [1]. In 2012, $M$. oleifera leaves were approved by the Chinese Ministry of Health as a new resource food. M. oleifera is also an essential traditional herb used to treat inflammation, diabetes, hypertension, anemia, hypoimmunity, and other diseases [2-5]. There are some flavonoid pigments, such as alkaloids, kaempferol, rhamnose, and isoquercitrin, and various antioxidant compounds, such as ascorbic acid, flavonoids, phenols, and carotenoids, which occur naturally in M. oleifera [6] and may contribute to the anti-inflammatory activity of $M$. oleifera. Additionally, it has been previously reported that some active components in M. oleifera mitigate some chronic inflammation [7]. However, there have been few reports on the improvement and alleviation of inflammation by $M$. oleifera polyphenols.

Inflammatory bowel disease (IBD), including ulcerative colitis (UC) and Crohn's disease (CD) [8], is a chronic recurrent disease of the intestine [9]. IBD is exceptionally harmful and is associated with an increased risk for colon cancer [10]. IBD is affected by diets such as high-fat diets, and the incidence is high in some developed countries, such as Europe [8]. Recently, the incidence of IBD in Asia has increased rapidly due to the improvements in living conditions and the adoption of a Western lifestyle. The pathogenesis of IBD is not yet fully clear; it is generally 
considered to be related to the environment, infection, genetics, psychology, and immunity. In addition, the intestinal mucosal immune system plays an essential role in the pathogenesis of IBD [11]. Several studies have shown that there is a high level of activated transcription factorkappa $B$ in the intestinal mucosa of IBD patients, and nuclear transcription factor kappa $\mathrm{B}(\mathrm{NF}-\kappa \mathrm{B})$ is considered to be one of the most critical signaling pathways in IBD. High expression of NF- $\kappa \mathrm{B}$ can enhance the release of proinflammatory cytokines, such as tumor necrosis factor- $\alpha$ (TNF- $\alpha$ ) and interleukin-6 (IL-6), which leads to colonic tissue damage [12]. It has been suggested that the activation of NF- $\kappa$ B plays a crucial role in the regulation of immune and inflammatory responses, and thus inhibiting the NF- $\kappa \mathrm{B}$ signaling pathway is a novel therapeutic strategy in IBD treatment research.

To date, there are no ideal drugs for the treatment of IBD. Existing treatments mainly include resection and relief medication, such as 5-aminosalicylic (5-ASA), corticosteroids, immunomodulatory drugs, and biological agents. However, long-term administration of these drugs has various side effects, including drug resistance and drug tolerance [13]. Therefore, it is necessary to find natural treatments with fewer side effects. Although M. oleifera has been reported to exhibit various bioactivities, knowledge about the effects of $M$. oleifera polyphenol extract (MOPE) on gut health is limited. The present study aimed to evaluate the effects of MOPE in an established model of acute ulcerative colitis induced with dextran sulfate sodium (DSS) and further explore its underlying mechanisms.

\section{Materials and Methods}

2.1. Preparation of MOPE. Dried M. oleifera leaves (Yunnan Shenbaofu Technology Development Co., Ltd., Dehong, China) were crushed and then extracted by the ultrasoundassisted method as described by Fei et al. [14]. The extraction process was performed three times under the same conditions with $70 \%$ ethanol as the solvent, a solid/solvent ratio of $1 / 30$, and $250 \mathrm{~W}$ of ultrasonic power for $20 \mathrm{~min}$. These three extraction supernatants were combined and passed through a D101 macroporous resin, followed by elution with an $80 \%$ ethanol solution to obtain an ethanol eluate. The eluate was vacuum freeze-dried to obtain the MOPE.

\subsection{Ultrahigh-Performance Liquid Chromatography Quad-} rupole Time-of-Flight Tandem Mass Spectrometry (UPLCQTOF-MS/MS). The composition of the MOPE was analyzed using a UPLC-QTOF-MS/MS system (XEVO G2-S QTOF-MS, Waters, USA). The chromatographic conditions were as follows: ACQUITY UPLC HSS T3 C18 $(2.1 \times 100 \mathrm{~mm}, 1.8 \mu \mathrm{m})$; mobile phase A $(0.1 \%$ formic acidwater), mobile phase $B$ (acetonitrile); flow rate: $0.5 \mathrm{~mL} \cdot \mathrm{min}^{-1}$; column temperature: $40^{\circ} \mathrm{C}$; and analysis time $13 \mathrm{~min}$. The sample was dissolved in $50 \%$ aqueous methanol, and the supernatant was used for analysis. The gradient elution was carried out as follows: $0 \mathrm{~min}, 10 \% \mathrm{~B} ; 6 \mathrm{~min}, 40 \%$ B; $6.2 \mathrm{~min}, 60 \% \mathrm{~B}$; $8.5 \mathrm{~min}, 90 \% \mathrm{~B}$; $10.5 \mathrm{~min}, 90 \% \mathrm{~B}$; and
$10.6 \mathrm{~min}, 10 \% \mathrm{~B}$. The mass spectrometry conditions were as follows: ESI: negative ion mode; scan range: $100-1200 \mathrm{Da}$; source temperature: $100^{\circ} \mathrm{C}$; desolvation temperature: $350^{\circ} \mathrm{C}$; desolvation gas flow: $700 \mathrm{~L} \cdot \mathrm{h}^{-1}$; LockSpray: leucine enkephalin (5-Leucine) enkephalin (LE); capillary: $2.8 \mathrm{kV}$; and sampling cone: $40 \mathrm{~V}$.

2.3. Animal Treatments. The animal experiments were performed according to international guidelines. The protocol was approved by the Animal Care and Use Committee of Yunnan Agricultural University (No. YNAU-2017-011). Six-week-old male C57BL/6 mice (18-20g, Liaoning Changsheng Biotechnology Co. Ltd., Liaoning, China) were maintained on a $12 / 12 \mathrm{~h}$ light/dark cycle at $25 \pm 1^{\circ} \mathrm{C}$ and $55 \%$ humidity. All mice were housed 5/cage and had free access to standard mouse feed and tap water. The mice were allowed to acclimate for one week before the study began.

2.4. Induction of Colitis and MOPE Treatment. Colitis was induced with dextran sulfate sodium (DSS, 36000-50000, MP Biomedicals, USA, 2160110). The mice were randomly divided into five groups ( $n=10$ per group): the control group was given ultrapure (UP) water for 14 days; the DSS group was given UP water for the first seven days and then $3 \%$ DSS for the following seven days; the 5-aminosalicylic acid (5-ASA, positive control) group and the MOPE groups $(50 \mathrm{mg} / \mathrm{kg}$ and $200 \mathrm{mg} / \mathrm{kg}$, respectively) were given 5 -ASA and MOPE individually for 14 days and were simultaneously given the water containing 3\% DSS starting on the eighth day (Figure 1(a)).

2.5. Evaluation of the Disease Activity Index (DAI). Mice were monitored daily for the development of colitis based on body weight changes, gross rectal bleeding, and stool consistency. The disease activity index (DAI) scores were measured for each animal according to a previously described method [15]. The details of the DAI grading standards are listed in the Supplementary Materials (Table S1).

2.6. Histopathology. The colon tissues $1 \mathrm{~cm}$ from the distal colon were rinsed with ice-cold PBS, fixed in $10 \%(\mathrm{v} / \mathrm{v})$ neutral formalin, embedded in paraffin, cut into $5 \mu \mathrm{m}$ slices (Leica, RM2126RT, Germany), and stained with hematoxylin and eosin (H\&E). Histological analysis was performed according to previously described methods [16]. A detailed description of the score standards is listed in the Supplementary Materials (Table S2).

2.7. Immunohistochemistry. The tissue slices were deparaffinized by xylene for $0.5-1 \mathrm{~h}$ and rehydrated sequentially with $100 \%, 100 \%, 95 \%$, and $80 \%$ ethanol and water for $5 \mathrm{~min}$ each. Then, the slices were immersed in methanol containing $3 \%$ hydrogen peroxide, followed by high-pressure antigen repair. The slides were incubated with primary antibodies against $\mathrm{CD}^{+}, \mathrm{CD}_{177^{+}}$, and $\mathrm{F} 4 / 80^{+}$(Abcam, Cambridge, MA) overnight and then incubated with the appropriate 


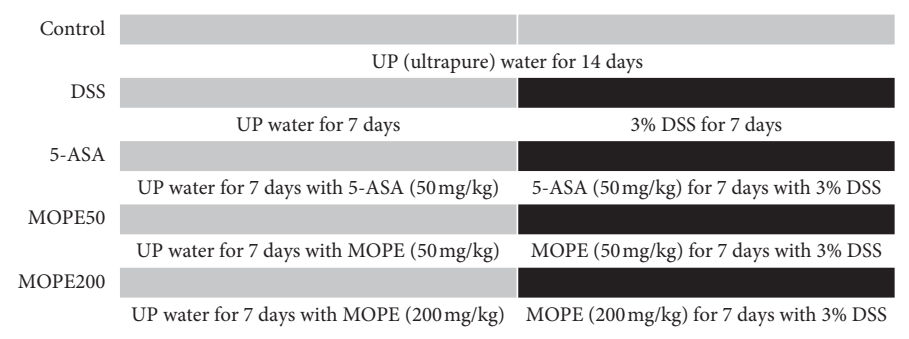

(a)

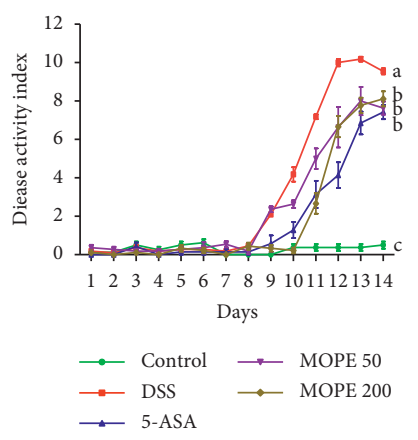

(c)

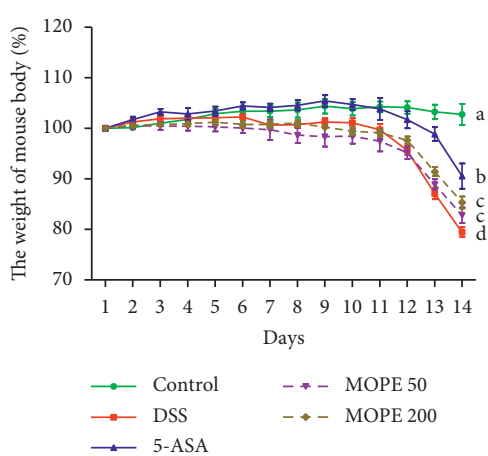

(b)

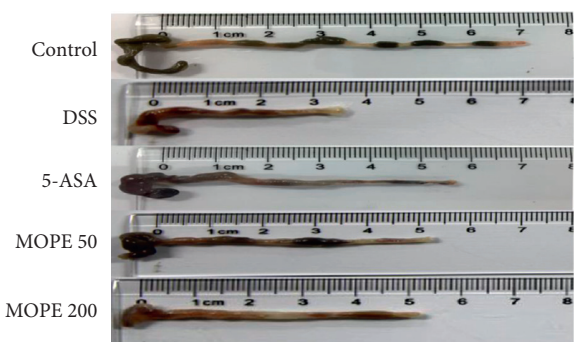

(d)

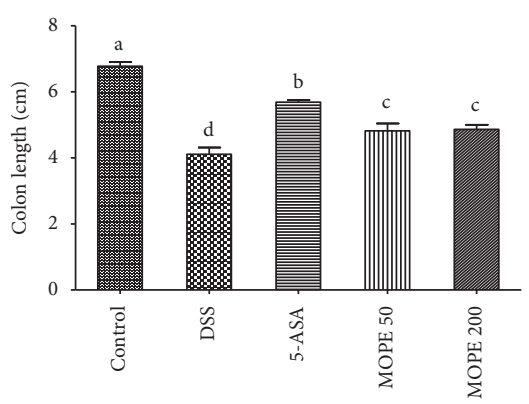

(e)

Figure 1: MOPE ameliorated DSS-induced colitis symptoms in mice. (a) The experimental design ( $n=10$ per group). (b) Body weight change in mice. (c) Disease activity index (DAI) scores. (d) Representative images of the mouse colon. (e) Colon lengths. The data are presented as the mean \pm SEM. Values with different letters differ significantly $(p<0.05)$.

secondary antibodies, followed by diaminobenzidine (DAB) color development, hematoxylin staining, and xylene clearing. Immunostained tissue slices were visualized under a microscope (Olympus, Japan).

\subsection{Determination of Serum Inflammatory Factors. At the} end of the experiment, serum samples were prepared and subjected to enzyme-linked immunosorbent assay (ELISA) to determine serum levels of IL- 6 and TNF- $\alpha$ using ELISA kits from BD Pharmingen. The procedure was performed according to the instructions.

2.9. Western Blotting. The colon tissues were homogenized and lysed in RIPA buffer (Solarbio) containing PMSF (100: 1) for $15 \mathrm{~min}$. After centrifugation $\left(4^{\circ} \mathrm{C}, 15000 \mathrm{rpm}, 10 \mathrm{~min}\right)$, the supernatant was collected, and the protein concentration was quantified using BCA protein assay reagent (Beyotime). The protein lysates ( $50 \mu \mathrm{g}$ protein) were separated by $10 \%$ sodium dodecyl sulfate-polyacrylamide gel electrophoresis and transferred to PVDF membranes (Immobilon). After being blocked with $5 \%$ bovine serum albumin (BSA) solution, the membranes were incubated with primary antibodies against TNF- $\alpha, \mathrm{NF}-\kappa \mathrm{B} p 65, \mathrm{I} \kappa \mathrm{B} \alpha$, and phosphorylated $\mathrm{I} \kappa \mathrm{B} \alpha$, followed by the appropriate secondary antibodies. Then, the protein-antibody complexes were developed with ECL Luminol reagent (Santa Cruz Biotechnology) and visualized.

2.10. Statistical Analysis. GraphPad Prism software version 5.0 was used to graph the results. The data are presented as the mean \pm SEM. Statistical analysis was performed by SPSS 19.0. The differences between multiple groups were analyzed by one-way analysis of variance (ANOVA), followed by 
Duncan's test. The results were considered statistically significant when $p<0.05$.

\section{Results}

3.1. UPLC-QTOF-MS/MS Analysis of MOPE. Seven compounds, including chlorogenic acid, rutin, isoquercitrin, astragalin, quercetin, luteolin, and kaempferitrin, which were known phenolic substances from MOPE, were analyzed by UPLC-QTOF-MS/MS. The retention times of these seven compounds were 1.57, 2.75, 2.92, 3.37, 4.74, 5.68, and $7.70 \mathrm{~min}$, respectively. The relative quantities were $4.66 \%$, $1.88 \%, 3.12 \%, 0.93 \%, 5.94 \%, 1.30 \%$, and $7.76 \%$, respectively (Figure 2).

3.2. MOPE Ameliorated Colitis Symptoms in Mice. The mice were treated with a low $(50 \mathrm{mg} / \mathrm{kg})$ or a high dose $(200 \mathrm{mg} / \mathrm{kg}$ ) of MOPE for 2 weeks (Figure 1(a)). Bodyweights and DAI scores of the mice were monitored every day throughout the experiment. The DSS-only treatment group exhibited severe bodyweight loss, while treatment with 5 -ASA (positive control) and $50 \mathrm{mg} / \mathrm{kg}$ and $200 \mathrm{mg} / \mathrm{kg}$ MOPE significantly alleviated weight loss (Figure 1(b)). Additionally, mice in the DSS group exhibited high DAI scores, while 5-ASA and MOPE treatment significantly decreased the DAI scores compared to those of the DSS group (Figure 1(c)). These results indicated that 5-ASA and MOPE treatments alleviated colonic inflammatory symptoms in mice.

The length of the colon is an important indicator for indirectly assessing the severity of colitis. In this study, fecal residues in the colons of control mice were normal and granular. The DSS group was induced to develop colitis, exhibiting hyperemic colons and very severe colon shortening. Furthermore, fecal residue in the colon in the 5-ASA group and MOPE groups was loose and less hyperemic (Figure 1(d)). 5-ASA and MOPE treatment significantly prevented the colonic shortening compared to that of the DSS-treated group (Figure 1(e)).

3.3. MOPE Ameliorated Colonic Injury in Mice with DSSInduced Colitis. The histological examination of colonic tissue from healthy mice revealed that the epithelial cells and crypts were structurally intact, the glands were arranged neatly, and there was no inflammatory cell infiltration (Figure 3(a)). In contrast, in the DSS-treated group, the lamina propria was damaged, the glands were destroyed and arranged irregularly, the epithelial cells and crypt structures were destroyed, and a large number of inflammatory cells infiltrated the muscle floor (Figure 3(b)). This damage was significantly alleviated after treatment with 5-ASA and MOPE (Figures 3(c)-3(f)).

3.4. MOPE Attenuated the Infiltration of Inflammatory Cells in Mice with DSS-Induced Colitis. The expression of $\mathrm{CD}^{+}$, $\mathrm{CD} 177^{+}$, and $\mathrm{F} 4 / 80^{+}$in the distal colon was measured by immunohistochemistry to detect $\mathrm{T}$ cell, neutrophil, and macrophage infiltration, respectively. The results indicated that T cells, neutrophils, and macrophages were increased in DSS-treated mice compared to normal mice, indicating that these inflammatory cells infiltrated the colonic injury area. 5ASA and MOPE treatment reduced the infiltration of T cells, neutrophils, and macrophages (Figure 4).

3.5. MOPE Decreased the Cytokine Levels in Mice with DSSInduced Colitis. IL- 6 and TNF- $\alpha$ are two typical inflammatory cytokines. The serum levels of IL- 6 and TNF- $\alpha$ were measured in the present study. Compared with those of the control group, substantial increases in IL-6 (Figure 5(a)) and TNF- $\alpha$ (Figure 5(b)) levels were observed in the DSS group $(p<0.001)$. However, MOPE treatment significantly reduced the serum levels of IL-6 $(p<0.01)$ and TNF- $\alpha$ $(p<0.05)$, and the effect was the same as that of 5-ASA (positive control). Moreover, MOPE treatment significantly inhibited the protein expression of TNF- $\alpha$ in colon tissue after DSS-induced colitis (Figures 5(c) and 5(d)).

\subsection{MOPE Modulated Inflammation-Related Signaling} Proteins. The NF- $\kappa \mathrm{B}$ signaling pathway strongly influences the pathogenesis of colitis. In the present study, the protein expression of NF- $\kappa \mathrm{B} p 65$ in the nucleus and $\mathrm{p}-\mathrm{I} \kappa \mathrm{B} \alpha$ in the colon tissue of DSS model mice was higher than that of control mice, and the protein expression of $\mathrm{I} \kappa \mathrm{B} \alpha$ was decreased (Figures $5(\mathrm{~d})-5(\mathrm{~g})$ ). This result indicated that the $\mathrm{NF}-\kappa \mathrm{B}$ signaling pathway was activated in the colonic mucosal. After treatment with MOPE, the protein expression of $\mathrm{NF}-\kappa \mathrm{B} p 65$ and $\mathrm{p}-\mathrm{I} \kappa \mathrm{B} \alpha$ was significantly downregulated, and the expression of $\mathrm{I} \kappa \mathrm{B} \alpha$ was upregulated. These results suggested that the anti-inflammatory effects of MOPE were related to inhibiting NF- $\kappa$ B pathway activation.

\section{Discussion}

M. oleifera has been reported to have beneficial effects on various diseases, including diabetes, hypertension, hyperlipidemia, and other chronic inflammation. There are some individual bioactive components in $M$. oleifera extracts that have potential preventive and therapeutic effects on inflammation [17]. Studies have shown that extracts of M. oleifera inhibit the production of $\mathrm{NO}$ and proinflammatory cytokines in LPS-induced macrophages [7, 18]. The pods of $M$. oleifera can inhibit the LPS-induced expression of IL- 6 and TNF- $\alpha$ and inhibit LPS-induced I $\kappa \mathrm{B}$ activation [19]. The ethyl acetate extract of $M$. oleifera can significantly inhibit LPS-induced TNF- $\alpha$, IL-6, and IL-8 production in human monocyte-derived macrophages (MDMs). M. oleifera extracts effectively inhibit the expression of inflammatory mediators that may be related to the inhibition of the NF- $\kappa \mathrm{B}$ signaling pathway $[3,20]$. In addition, it has been reported that the hydroalcoholic extract of $M$. oleifera seeds can reduce acetic acid-induced colitis in rats [21]. Moreover, the isothiocyanate extracted from M. oleifera seeds can effectively alleviate DSS-induced colitis [22]. However, knowledge about the effects of MOPE on gut health is limited. Therefore, the present study aimed to 


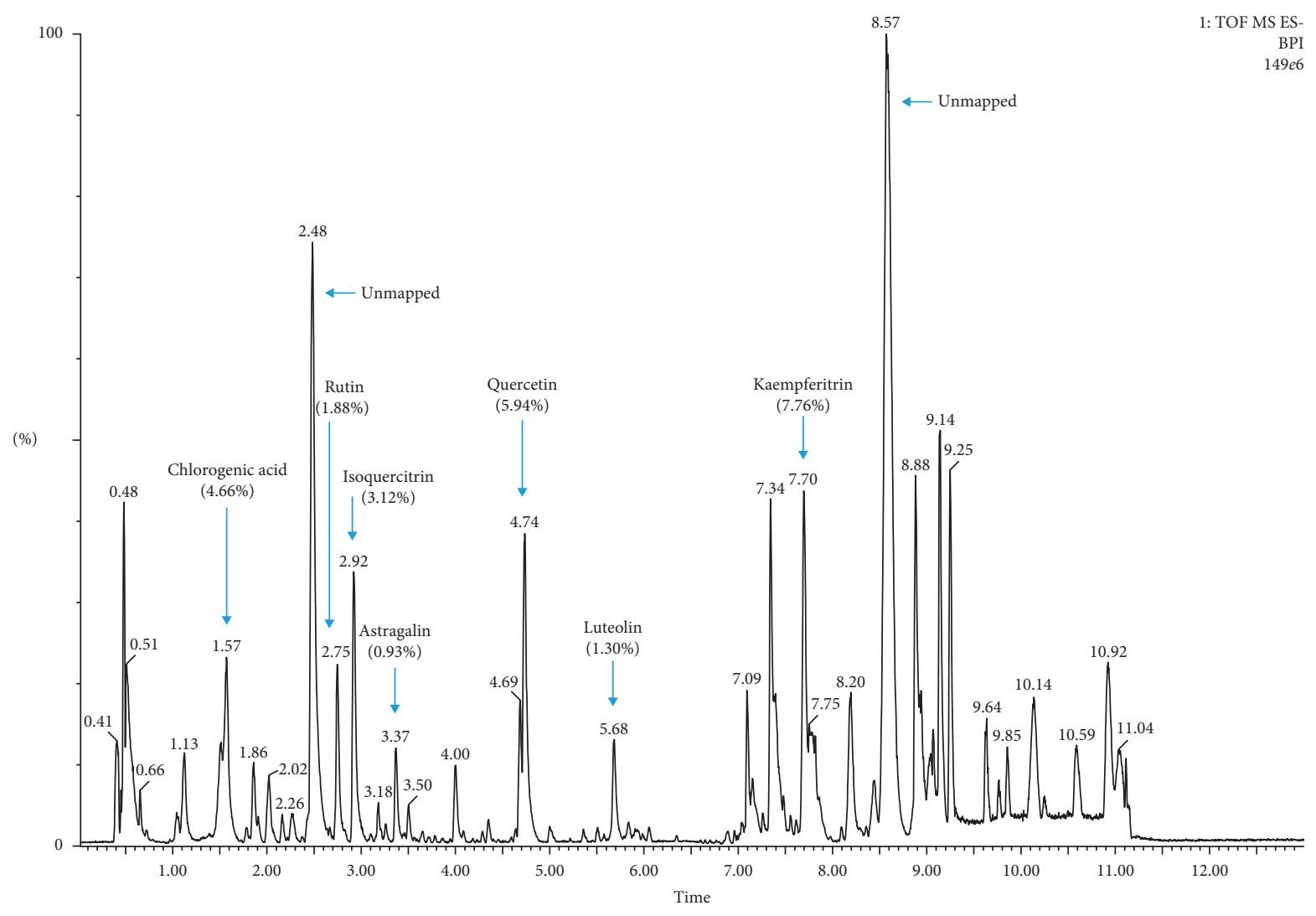

Figure 2: UPLC-QTOF-MS/MS chromatogram of MOPE.

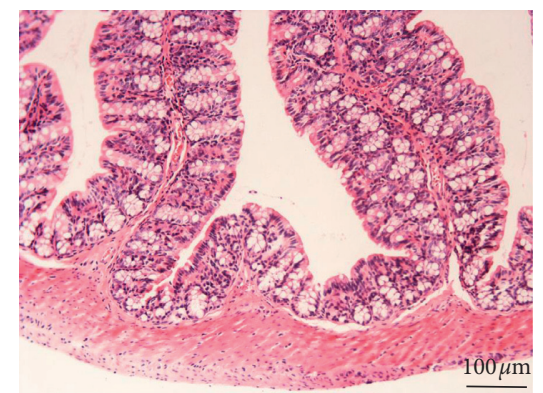

(a)

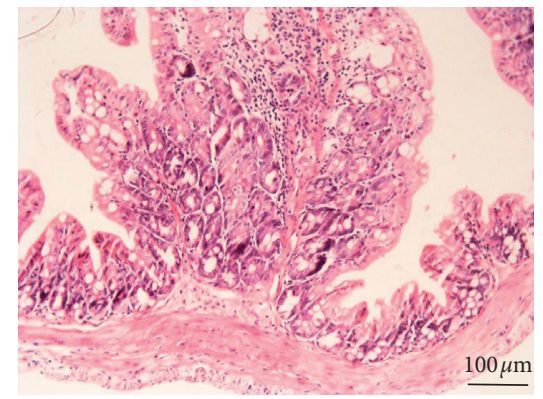

(d)

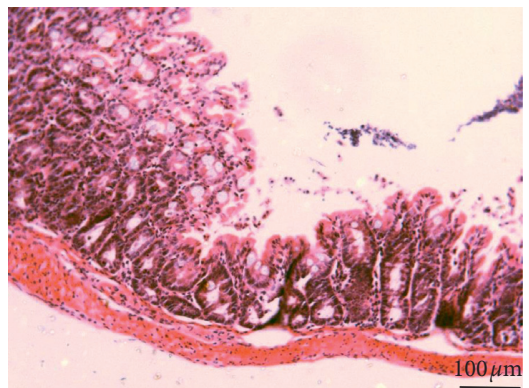

(b)

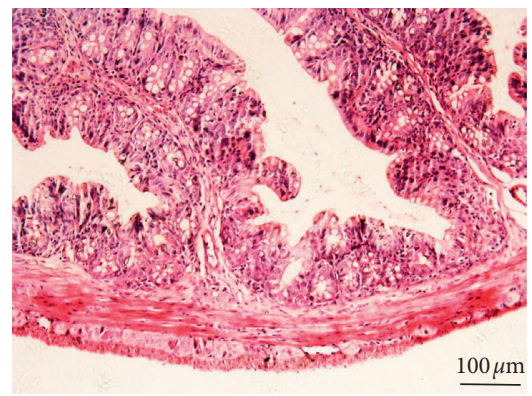

(e)

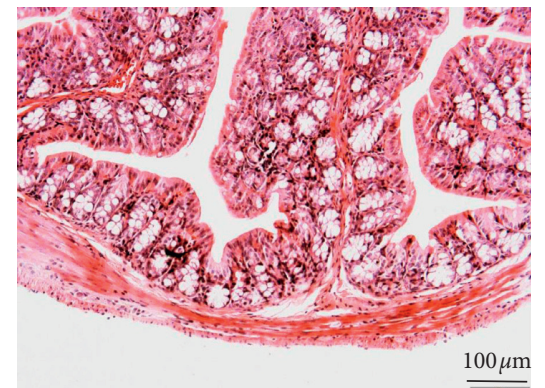

(c)

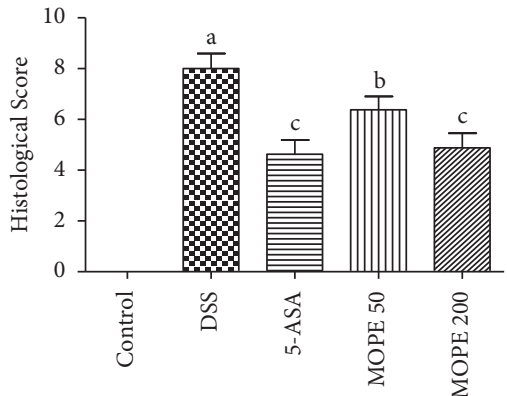

(f)

FIgURE 3: Effects of MOPE on the histopathological characterization of DSS-colitis mice. Representative HE-stained sections of the distal colonic tissues from the (a) control, (b) DSS, (c) 5-ASA (50 mg/kg), (d) MOPE (50 mg/kg), and (e) MOPE (200 mg/kg) groups. All images were acquired using 200× magnification. (f) Histological scores of colonic abnormalities. Values with different letters (a-c) differ significantly $(p<0.05)$. 


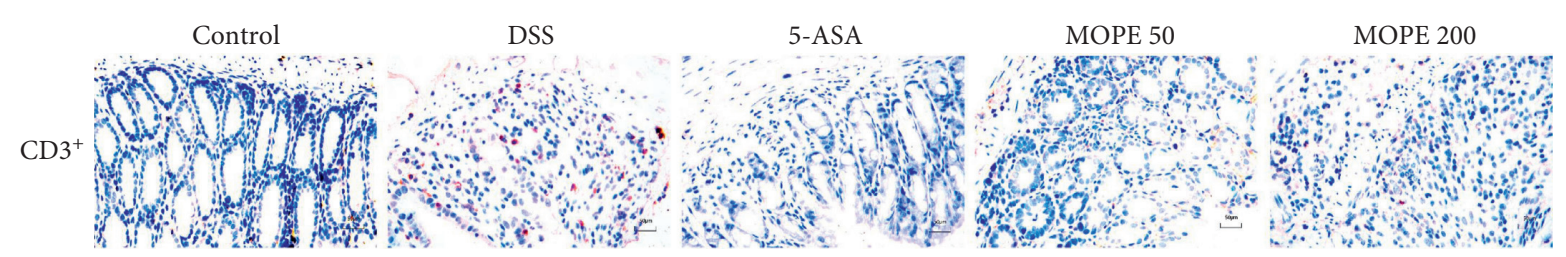

(a)
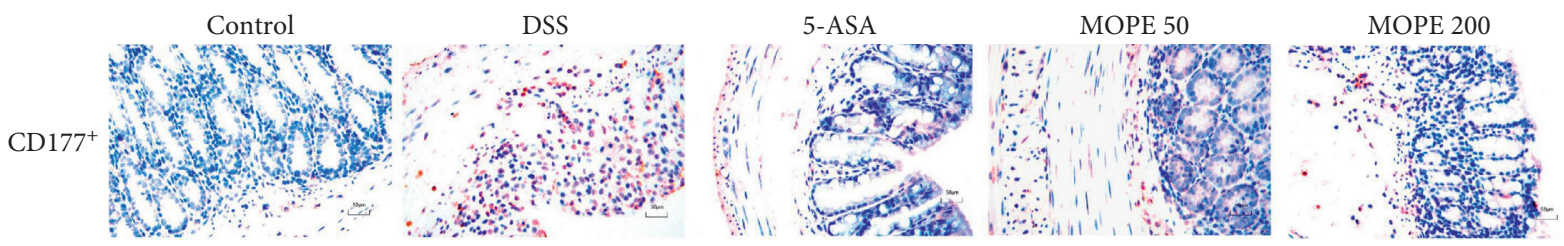

(b)

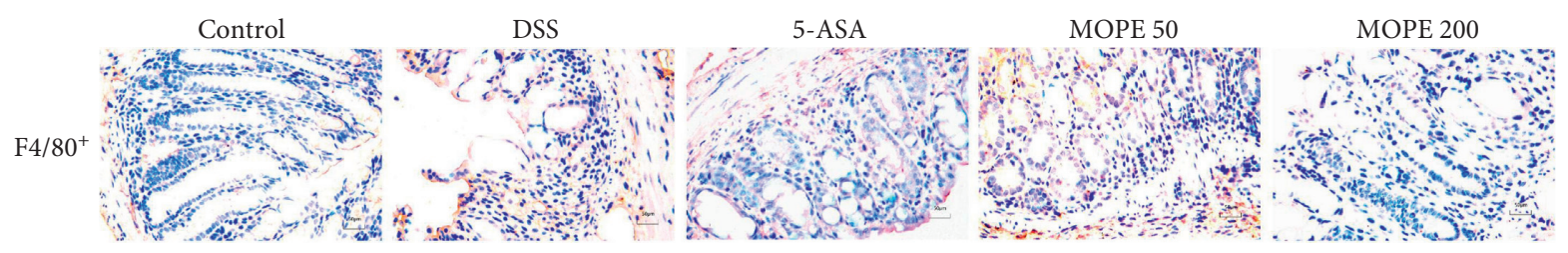

(c)

FIGURE 4: MOPE attenuated the infiltration of inflammatory cells in mice with DSS-induced colitis. Representative images of (a) CD3 ${ }^{+}$, (b) $\mathrm{CD}_{177^{+}}$, (c) and $\mathrm{F} 4 / 80^{+}$immunostaining in the distal colons of mice; original magnification: 400 $\times$.

investigate MOPE-mediated alleviation of DSS-induced colitis in mice.

Here, we showed that MOPE could significantly ameliorate the symptoms of DSS-induced colitis, including mitigating body weight loss, colonic tissue damage, the secretion of inflammatory cytokines, and the infiltration of inflammatory cells. IL- 6 and TNF- $\alpha$ are proinflammatory cytokines that mediate inflammatory responses in the UC model. Excessive secretion of these inflammatory cytokines can cause colitis [15]. Many studies have reported that the levels of IL- 6 and TNF- $\alpha$ in IBD mice are increased [23-25]. IL-6 is an interleukin produced by a variety of cells and is closely related to inflammation and the immune response. IL- 6 stimulates neutrophil chemotaxis and causes tissue destruction in the colon. Some studies have found that IL- 6 is elevated in many IBDs and may be associated with the NF- $\kappa \mathrm{B}$ signaling pathway $[26,27]$. TNF- $\alpha$, a cytokine with multiple effects that is produced by activated $\mathrm{T}$ cells, plays an essential role in the pathogenesis of colitis by triggering the accumulation and activation of leukocytes. TNF- $\alpha$ overexpression is vital for the pathogenesis of the intestinal mucosa $[28,29]$. In the present study, DSS induced an increase in TNF- $\alpha$ and IL- 6 in mice, whereas MOPE treatment significantly reduced the increases in both inflammatory factors.

$\mathrm{NF}-\kappa \mathrm{B}$ is a classic signaling pathway associated with inflammation. Several studies have shown that some natural compounds, such as caffeic acid, blueberry polyphenols, and Aster glehni extract, can improve UC by inhibiting the NF$\kappa \mathrm{B}$ pathway $[25,30-32]$. It was shown that the ethyl acetate extract of $M$. oleifera inhibited NF- $\kappa$ B p65 in RAW 264.7 cells, and it exerted anti-inflammatory effects by upregulating the expression of $\mathrm{I} \kappa \mathrm{B}$ inhibitor $(\mathrm{I} \kappa \mathrm{B} \alpha)$ and blocking the nuclear translocation of NF- $\kappa \mathrm{B}$ [3]. NF- $\kappa \mathrm{B}$ family members are retained in the cytoplasm bound to a class of inhibitory proteins termed $\mathrm{I} \kappa \mathrm{Bs}[33]$. When cells are activated, $\mathrm{I} \kappa \mathrm{B}$ is phosphorylated by $\mathrm{I} \kappa \mathrm{B}$ kinase (IKK) and degraded, after which the released NF- $\kappa \mathrm{B}$ translocates to the nucleus and activates transcription. In other words, phosphorylation of $\mathrm{I} \kappa \mathrm{B}(\mathrm{p}-\mathrm{I} \kappa \mathrm{B})$ is a crucial step in NF- $\kappa \mathrm{B}$ activation, which leads to activation of the NF- $\kappa \mathrm{B}$ signaling pathway [34]. The increase in TNF- $\alpha$-related indicators is an essential manifestation of NF- $\kappa \mathrm{B}$ pathway activation. Many treatments target TNF- $\alpha$, and some related drugs, including TNF- $\alpha$ blockers, have been successfully used in the treatment of patients with IBD $[31,35]$. For these reasons, the proteins related to the NF- $\kappa \mathrm{B}$ signaling pathways were investigated in the present study. Our results showed that the protein expression of NF- $\kappa \mathrm{B} p 65$ in the nucleus and $\mathrm{p}-\mathrm{I} \kappa \mathrm{B} \alpha$ and TNF- $\alpha$ in colon tissue of DSS model mice was higher than that of control mice, and the expression of $\mathrm{I} \kappa \mathrm{B} \alpha$ was decreased. This result indicated that the NF- $\kappa$ B pathway was activated in the colonic mucosa. After MOPE administration, the protein expression of NF- $\kappa \mathrm{B} p 65$ and $\mathrm{p}-\mathrm{I} \kappa \mathrm{B} \alpha$ was downregulated, and the expression of $\mathrm{I} \kappa \mathrm{B} \alpha$ was upregulated. Furthermore, MOPE treatment inhibited the high expression of TNF- $\alpha$ in colon tissue. These results indicate that the anti-inflammatory effects of MOPE may be related to the inhibition of the NF- $\kappa \mathrm{B}$ signaling pathway.

In the present study, MOPE is a mixed extract. The main phenolic substances in MOPE are kaempferol, quercetin, chlorogenic acid, isoquercitrin, rutin, and luteolin, as determined by UPLC-QTOF-MS/MS analysis, which is consistent with the results of the study by Zhu et al. [36]. In the future, we will focus on the separation and purification of $M$. oleifera polyphenols to determine which phenolic substance has antiinflammatory or multicomponent synergistic effects. 


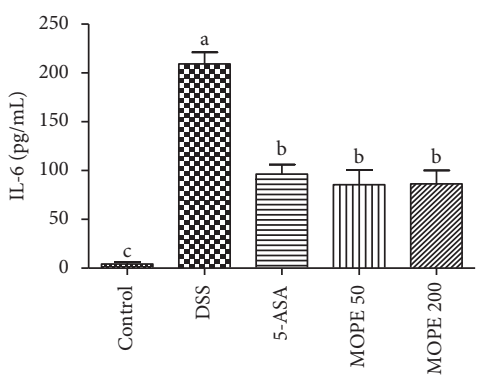

(a)

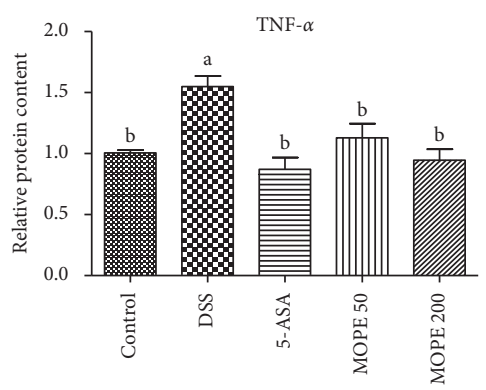

(d)

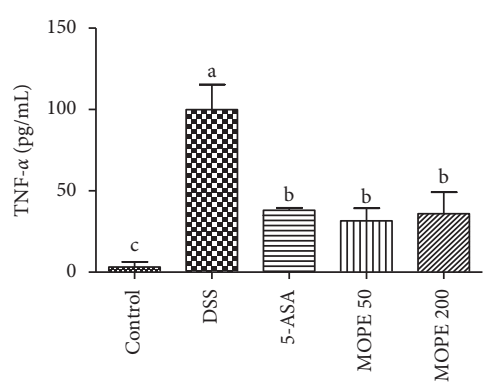

(b)

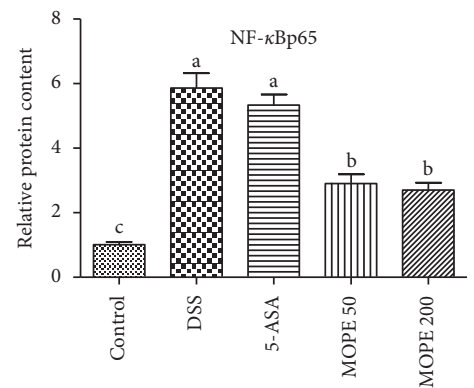

(e)

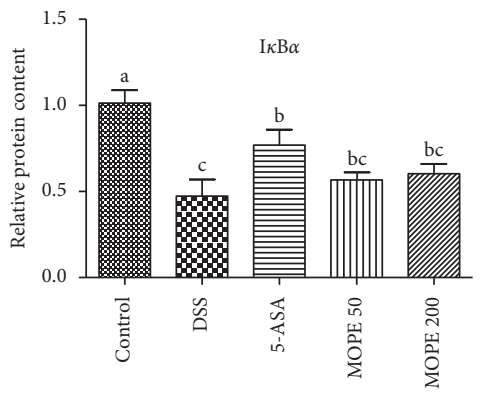

(g)

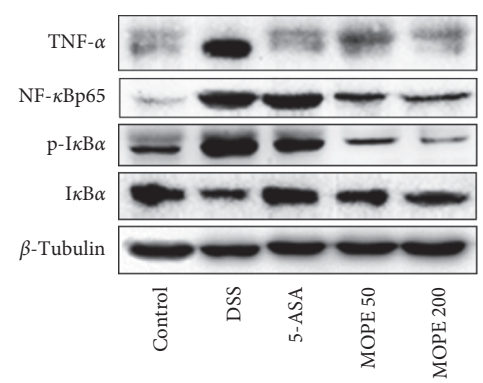

(c)

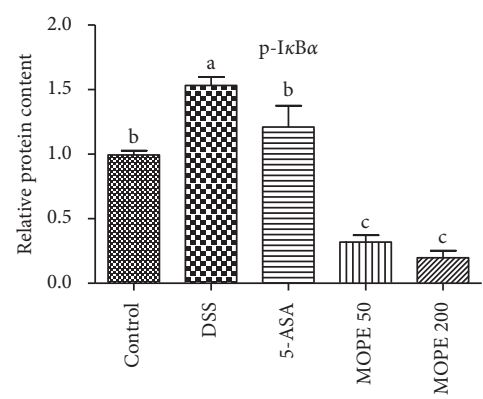

(f)

FIGURE 5: MOPE modulated cytokines and inflammation-related signaling proteins in mice with DSS-induced colitis. (a) The serum IL-6 level. (b) The serum TNF- $\alpha$. (c) Western blot analysis of key signaling proteins in colonic tissue. (d) Quantitative analysis of TNF- $\alpha$ protein levels in colonic tissue. (e) Quantitative analysis of NF- $\kappa \mathrm{B} p 65$ protein levels. (f) Quantitative analysis of (p)-I $\kappa \mathrm{B} \alpha$ protein levels. (g) Quantitative analysis of $\mathrm{I} \kappa \mathrm{B} \alpha$ protein levels. Values with different letters $(\mathrm{a}-\mathrm{c})$ differ significantly $(p<0.05)$.

In conclusion, our research shows that MOPE can alleviate DSS-induced colitis, including mitigating body weight loss, colon shortening, the secretion of inflammatory cytokines, colon tissue damage, and inflammatory cell infiltration. Further study examination shows that MOPE may alleviate colitis by inhibiting NF- $\kappa \mathrm{B}$ signaling pathway activation. These results indicate that $M$. oleifera can be developed as a potential health food for preventing colitis.

\section{Data Availability}

The data to support the findings of this study are included within the article. Other data used to support the findings of this study are available from the corresponding author upon request.

\section{Conflicts of Interest}

The authors declare that there are no conflicts of interest regarding the publication of this paper.

\section{Authors' Contributions}

Yunjuan Zhang and Lei Peng contributed equally to this article.

\section{Acknowledgments}

This work was supported by the Ministry of Agriculture Tropical Crop Technology Pilot Demonstration Project " $M$. oleifera product processing demonstration" (K2500053), "Deep Processing and Technical Demonstration of Moringa, Macadamia and Tropical Fruits" (18190026), and Personnel Training Project on Academic and Technical Leaders of Yunnan Province (2018HB040).

\section{Supplementary Materials}

The details of the DAI grading standards are listed in the Supplementary Materials (Table S1). A detailed description of 
the Histological Scores of Colon Damage is listed in the Supplementary Materials (Table S2). (Supplementary Materials)

\section{References}

[1] C. Waterman, D. M. Cheng, P. Rojas-Silva et al., "Stable, water extractable isothiocyanates from Moringa oleifera leaves attenuate inflammation in vitro," Phytochemistry, vol. 103, pp. 114-122, 2014.

[2] I. Matic, A. Guidi, M. Kenzo, M. Mattei, and A. Galgani, "Investigation of medicinal plants traditionally used as dietary supplements: a review on Moringa oleifera," Journal of Public Health in Africa, vol. 9, no. 3, pp. 191-199, 2018.

[3] P. Arulselvan, W. S. Tan, S. Gothai et al., "Anti-Inflammatory potential of ethyl acetate fraction of Moringa oleifera in downregulating the NF-kappaB signaling pathway in lipopolysaccharide-stimulated macrophages," Molecules, vol. 21, no. 11, Article ID e1452, 2016.

[4] M. J. Tuorkey, "Effects of Moringa oleiferaaqueous leaf extract in alloxan induced diabetic mice," Interventional Medicine and Applied Science, vol. 8, no. 3, pp. 109-117, 2016.

[5] J. Xie, Y. Wang, W. W. Jiang et al., "Moringa oleifera leaf petroleum ether extract inhibits lipogenesis by activating the AMPK signaling pathway," Frontiers in Pharmacology, vol. 9, Article ID 1447, 2018.

[6] F. Anwar, S. Latif, M. Ashraf, and A. H. Gilani, "Moringa oleifera: a food plant with multiple medicinal uses," Phytotherapy Research, vol. 21, no. 1, pp. 17-25, 2007.

[7] A. Jaja-Chimedza, B. L. Graf, C. Simmler et al., "Biochemical characterization and anti-inflammatory properties of an isothiocyanate-enriched moringa (Moringa oleifera) seed extract," PLoS One, vol. 12, no. 8, 2017.

[8] J. Ni, G. D. Wu, L. Albenberg, and V. T. Tomov, "Gut microbiota and IBD: causation or correlation?" Nature Reviews Gastroenterology \& Hepatology, vol. 14, no. 10, pp. 573-584, 2017.

[9] G. Trallori, D. Palli, C. Saieva et al., "A population-based study of inflammatory bowel disease in Florence over 15 years (1978-92)," Scandinavian Journal of Gastroenterology, vol. 31, no. 9, pp. 892-899, 1996.

[10] M. S. Nadeem, V. Kumar, F. A. Al-Abbasi, M. A. Kamal, and F. Anwar, "Risk of colorectal cancer in inflammatory bowel diseases," Seminars in Cancer Biology, vol. 64, pp. 51-60, 2020.

[11] I. Atreya, R. Atreya, and M. F. Neurath, "NF- $\kappa$ B in inflammatory bowel disease," Journal of Internal Medicine, vol. 263, no. 6, pp. 591-596, 2008.

[12] E. Giner, I. Andújar, M. C. Recio, J. L. Ríos, J. M. CerdáNicolás, and R. M. Giner, "Oleuropein ameliorates acute colitis in mice," Journal of Agricultural and Food Chemistry, vol. 59, no. 24, pp. 12882-12892, 2011.

[13] C. Sostres, C. J. Gargallo, M. T. Arroyo, and A. Lanas, "Adverse effects of non-steroidal anti-inflammatory drugs (NSAIDs, aspirin and coxibs) on upper gastrointestinal tract," Best Practice \& Research Clinical Gastroenterology, vol. 24, no. 2, pp. 121-132, 2010.

[14] F. Pei, H. Tao, L. Cai et al., "Optimization of polyphenols from Moringa oleifera Lam. leaves by ultrasound-assisted extraction using response surface methodology and their antioxidant activities," Food Science, vol. 37, no. 20, pp. 24-30, 2016, in Chinese.

[15] S.-H. Kang, Y.-D. Jeon, K.-H. Moon et al., "Aronia berry extract ameliorates the severity of dextran sodium sulfateinduced ulcerative colitis in mice," Journal of Medicinal Food, vol. 20, no. 7, pp. 667-675, 2017.
[16] W. Liu, Y. Zhang, B. Qiu et al., "Quinoa whole grain diet compromises the changes of gut microbiota and colonic colitis induced by dextran Sulfate sodium in C57BL/6 mice," Scientific Reports, vol. 8, no. 1, Article ID 14916, 2018.

[17] N. Kooltheat, R. Sranujit, P. Chumark, P. Potup, N. Laytragoon-Lewin, and K. Usuwanthim, "An ethyl acetate fraction of Moringa oleifera Lam. inhibits human macrophage cytokine production induced by cigarette smoke," Nutrients, vol. 6, no. 2, pp. 697-710, 2014.

[18] X. Kou, B. Li, J. B. Olayanju, J. M. Drake, and N. Chen, "Nutraceutical or pharmacological potential of Moringa oleifera Lam," Nutrients, vol. 10, no. 3, p. 343, 2018.

[19] C. Muangnoi, P. Chingsuwanrote, P. Praengamthanachoti, S. Svasti, and S. Tuntipopipat, "Moringa oleifera pod inhibits inflammatory mediator production by lipopolysaccharidestimulated RAW 264.7 murine macrophage cell lines," Inflammation, vol. 35, no. 2, pp. 445-455, 2012.

[20] M. T. Fard, P. Arulselvan, G. Karthivashan, S. K. Adam, and S. Fakurazi, "Bioactive extract from Moringa oleifera inhibits the pro-inflammatory mediators in lipopolysaccharide stimulated macrophages," Pharmacognosy Magazine, vol. 11, no. 4, pp. 556-563, 2015.

[21] M. Minaiyan, G. Asghari, D. Taheri, M. Saeidi, and S. NasrEsfahani, "Anti-inflammatory effect of Moringa oleifera Lam. seeds on acetic acid-induced acute colitis in rats," Avicenna Journal of Phytomedicine, vol. 4, no. 2, pp. 127-136, 2014.

[22] Y. Kim, A. G. Wu, A. Jaja-Chimedza et al., "Isothiocyanateenriched Moringa seed extract alleviates ulcerative colitis symptoms in mice," PLoS One, vol. 12, no. 9, 2017.

[23] Z. Zhang, X. Wu, S. Cao et al., "Chlorogenic acid ameliorates experimental colitis by promoting growth of Akkermansia in mice," Nutrients, vol. 9, no. 7, p. 677, 2017.

[24] B. Liu, T. Yang, L. Zeng et al., "Crude extract of Fuzhuan brick tea ameliorates DSS-induced colitis in mice," International Journal of Food Science \& Technology, vol. 51, no. 12, pp. 2574-2582, 2016.

[25] Z. Zhang, X. Wu, S. Cao et al., "Caffeic acid ameliorates colitis in association with increased Akkermansia population in the gut microbiota of mice," Oncotarget, vol. 7, no. 22, pp. 31790-31799, 2016.

[26] L.-C. Tong, Y. Wang, Z.-B. Wang et al., "Propionate ameliorates dextran sodium sulfate-induced colitis by improving intestinal barrier function and reducing inflammation and oxidative stress," Frontiers in Pharmacology, vol. 7, p. 253, 2016.

[27] S.-E. Jang, S.-M. Lim, J.-J. Jeong et al., "Gastrointestinal inflammation by gut microbiota disturbance induces memory impairment in mice," Mucosal Immunology, vol. 11, no. 2, pp. 369-379, 2018.

[28] S. J. van Deventer, "Immunology in medical practice. IV. Inflammatory bowel diseases: pathogenic starting points for specific therapy," Nederlands Tijdschrift Voor Geneeskunde, vol. 141, no. 41, pp. 1956-1959, 1997.

[29] F. Biasi, G. Leonarduzzi, P. I. Oteiza, and G. Poli, "Inflammatory bowel disease: mechanisms, redox considerations, and therapeutic targets," Antioxidants \& Redox Signaling, vol. 19, no. 14, pp. 1711-1747, 2013.

[30] M. Pervin, M. A. Hasnat, J.-H. Lim et al., "Preventive and therapeutic effects of blueberry (Vaccinium corymbosum) extract against DSS-induced ulcerative colitis by regulation of antioxidant and inflammatory mediators," The Journal of Nutritional Biochemistry, vol. 28, pp. 103-113, 2016.

[31] Z.-L. Zhang, H.-Y. Fan, M.-Y. Yang, Z.-K. Zhang, and K. Liu, "Therapeutic effect of a hydroxynaphthoquinone fraction on 
dextran sulfate sodium-induced ulcerative colitis," World Journal of Gastroenterology, vol. 20, no. 41, pp. 15310-15318, 2014.

[32] J.-H. Choi, K.-S. Chung, B.-R. Jin et al., “Anti-inflammatory effects of an ethanol extract of Aster glehni via inhibition of NF- $\kappa$ B activation in mice with DSS-induced colitis," Food \& Function, vol. 8, no. 7, pp. 2611-2620, 2017.

[33] X. Liu, S. Togo, M. Al-Mugotir et al., "NF-kappaB mediates the survival of human bronchial epithelial cells exposed to cigarette smoke extract," Respiratory Research, vol. 9, no. 1, p. $66,2008$.

[34] P. P. Tak and G. S. Firestein, "NF- $\kappa$ B: a key role in inflammatory diseases," Journal of Clinical Investigation, vol. 107, no. 1, pp. 7-11, 2001.

[35] J. S. Hyams, T. Lerer, A. Griffiths et al., "Long-term outcome of maintenance infliximab therapy in children with Crohn's disease," Inflammatory Bowel Diseases, vol. 15, no. 6, pp. 816-822, 2009.

[36] Y. Zhu, Q. Yin, and Y. Yang, "Comprehensive investigation of Moringa oleifera from different regions by simultaneous determination of 11 polyphenols using UPLC-ESI-MS/MS," Molecules, vol. 25, no. 3, Article ID e676, 2020. 